\title{
Brief Report \\ XDR-Pseudomonas aeruginosa Outside the ICU: Is There Still Place for Colistin?
}

\author{
Paola Del Giacomo ${ }^{1}$, Francesca Raffaelli ${ }^{1}$, Angela Raffaella Losito ${ }^{1} \mathbb{D}$, Barbara Fiori ${ }^{1}$ and Mario Tumbarello ${ }^{2,3, * \mathbb{D}}$ \\ 1 Dipartimento di Scienze di Laboratorio e Infettivologiche, Fondazione Policlinico Universitario A. Gemelli \\ IRCCS, 00168 Rome, Italy; paola.delgiacomo@policlinicogemelli.it (P.D.G.); \\ francesca.raffaelli@policlinicogemelli.it (F.R.); angelaraffaella.losito@policlinicogemelli.it (A.R.L.); \\ barbara.fiori@policlinicogemelli.it (B.F.) \\ 2 Dipartimento di Biotecnologie Mediche, Università degli Studi di Siena, 16, 53100 Siena, Italy \\ 3 UOC Malattie Infettive e Tropicali, Azienda Ospedaliero Universitaria Senese, 53100 Siena, Italy \\ * Correspondence: mario.tumbarello@unisi.it or mariotumb@gmail.com; Tel.: +39-0577-586572
}

check for updates

Citation: Del Giacomo, P.; Raffaelli, F.; Losito, A.R.; Fiori, B.; Tumbarello, M. XDR-Pseudomonas aeruginosa Outside the ICU: Is There Still Place for Colistin? Antibiotics 2022, 11, 193. https://doi.org/10.3390/ antibiotics11020193

Academic Editor: Luigi Principe

Received: 10 January 2022

Accepted: 29 January 2022

Published: 1 February 2022

Publisher's Note: MDPI stays neutral with regard to jurisdictional claims in published maps and institutional affiliations.

Copyright: () 2022 by the authors Licensee MDPI, Basel, Switzerland. This article is an open access article distributed under the terms and conditions of the Creative Commons Attribution (CC BY) license (https:// creativecommons.org/licenses/by/ $4.0 /)$.

\begin{abstract}
Background: Pseudomonas aeruginosa represents, among the nosocomial pathogens, one of the most serious threats, both for the severity of its clinical manifestations and its ability to develop complex profiles of resistance; Methods: we retrospectively collected the data of 21 patients admitted to a tertiary-care University Hospital of Rome with infections due to XDR-P. aeruginosa isolates during the second half of 2020; Results: in our institution, the percentage of XDR-P. aeruginosa isolates is $3.1 \%$. None of the patients was admitted to the intensive care unit at the moment of the infection's onset. Susceptibility to colistin was preserved in all the tested isolates. Rates of resistance to ceftolozane/tazobactam and ceftazidime/avibactam in these XDR strains were consistent; Conclusions: XDR-P. aeruginosa can be a threatening problem even outside the ICUs, especially in frail patients in wards with features of long-term acute care hospitals. In such a setting, ceftolozane/tazobactam and ceftazidime/avibactam should be administered with caution taking into account the microbiological susceptibility results. Colistin, even with its known safety and efficacy limits, could represent the only available therapeutic option due to its highly preserved susceptibility against XDR isolates of $P$. aeruginosa.
\end{abstract}

Keywords: Pseudomonas aeruginosa; extensively drug resistant (XDR); ceftolozane/tazobactam; ceftazidime/avibactam; colistin

\section{Introduction}

Pseudomonas aeruginosa (PA) is a common nosocomial pathogen worldwide and it represents one of the most challenging microorganisms to face in clinical practice due to its intrinsic resistance and its extraordinary ability to develop additional resistance through selection of chromosomal mutations and acquisition of resistance genes [1]. The isolation of extensively drug-resistant Pseudomonas aeruginosa strains (XDR-PA) seriously compromises the probability of receiving an appropriate initial antibiotic therapy, possibly leading to poor outcomes, particularly in the presence of severe infections.

\section{Methods}

We retrospectively collected, through the daily microbiological report of multidrugresistant (MDR) or extensively drug-resistant (XDR) isolates delivered by the Microbiology Laboratory, data of 21 patients admitted to a tertiary-care at the University Hospital of Rome (Fondazione Policlinico Universitario Agostino Gemelli IRCCS) with documented XDR-Pseudomonas aeruginosa isolates from 1 June to 31 December 2020. Patients were selected if they had documented true infections due to Pseudomonas aeruginosa strains nonsusceptible to at least one agent in all but two or fewer antimicrobial categories according to the established definition of XDR (anti-pseudomonal cephalosporins, anti-pseudomonal 
penicillin plus $\beta$-lactamase inhibitors, monobactams, anti-pseudomonal carbapenems, aminoglycosides, fluoroquinolones, phosphonic acid and polymyxins) [2]. Each patient was included only once, at the time of the XDR-PA infection onset. Infections were classified as XDR-PA bacteremia if blood cultures were positive for XDR-PA strain (with or without the same XDR-PA isolation from other sites) and there were clinical signs of systemic inflammatory response syndrome.

Two patients were excluded because they were not classified as infections but colonizations. Colonization was defined as the isolation of PA from any clinical specimens (except blood) in the absence of signs or symptoms of infections, and the patients had not received antimicrobial treatment related to the culture results.

Isolates were identified using a MALDI Biotyper (Bruker Daltonik GmbH, Leipzig, Germany).

The minimum inhibitory concentrations (MICs) of ceftazidime, cefepime, ceftazidime/ avibactam, ceftolozane/tazobactam, piperacillin-tazobactam, meropenem, imipenem, ciprofloxacin, amikacin, gentamicin and tobramycin were determined by a VITEK ${ }^{\circledR} 2$ system (bioMérieux) and, for colistin, when feasible, by commercial broth-microdilution antimicrobial susceptibility testing panels (MERLIN Diagnostica GmbH, Bornheim, Germany) according to EUCAST guidelines. Results were interpreted according to European Committee on Antimicrobial Susceptibility Testing (EUCAST) clinical breakpoints [3,4]. The NG-Test Carba 5 immunochromatographic assay (NG Biotech, Guipry, France) was used to detect the five main carbapenemases, i.e., KPC, OXA-48-like, NDM, VIM, and IMP [5].

Adequate empiric therapy was deemed an appropriate dosage regimen of one or more antibiotics to which the isolate was sensitive in vitro. An effective definitive therapy was defined as treatment with at least one effective anti-pseudomonal agent based on antimicrobial susceptibility testing. The timing to evaluate the appropriate initiation of a target antibiotic therapy was set at $48 \mathrm{~h}$ from the infection onset.

Clinical success was defined as the resolution of signs and symptoms of acute infection. Absence of recurrent infection was evaluated at 30 days following the onset of infection or at time of discharge from the hospital.

Microbiological failure was defined as isolation of Pseudomonas aeruginosa with the same susceptibility profile at the same site $\geq 7$ days after the target therapy initiation.

\section{Results}

Nineteen patients with XDR-PA infections were included in the study. The median age was 69 (IQR 64-80). Males comprised 68.4\%. No community-acquired infection was found; $11(57.9 \%)$ of the infections were classified as hospital-acquired infection and were inpatients belonging to medical wards at the time of the infection onset: $63.6 \%$ of the patients in the Pneumology and the Neurology/Neurological rehabilitation clinic. Eight $(42.1 \%)$ of the patients were assumed to have a healthcare-related infection. In five (26.3\%) cases, an admission in an Intensive Care Unit (ICU) in the previous 90 days was reported. Eight $(42.1 \%)$ patients had undergone surgical or invasive procedures in the previous 30 days. All the patients had at least one comorbidity, with a median Age-Adjusted Charlson Comorbidity index of 6.

In $16(84.2 \%)$ patients, a previous bacterial infection was documented. Among these, a previous PA infection was documented in six (31.6\%) patients. Prior isolation of MDR/XDR bacteria was reported in 11 (57.9\%) patients (Methicillin-resistant Staphylococcus aureus, Vancomycin-resistant Enterococcus, Carbapenem-resistant Enterobacterales, MDR/XDR-PA). Previous antibiotic therapy was reported in $15(78.9 \%)$ patients. Carbapenems were the most frequent prior antimicrobials reported (42.1\%), together with glycopeptides (42.1\%) and followed by piperacillin/tazobactam (36.8\%). In $14(73.7 \%)$ patients, prior antimicrobial use was as long as seven days or longer. In 12 (63.2\%) cases, the antimicrobial therapy consisted of multiple antibiotics (sequential or used in combinations). One or more invasive medical devices were present in $17(89.5 \%)$ of the patients: Foley catheter was present in $14(73.7 \%)$, tracheostomy in seven $(36.8 \%)$ of the patients, central venous catheter in five of the patients $(26.3 \%)$. 
True XDR-PA infections included, six (31.6\%) UTIs, five (26.3\%) BSIs, four $(21.1 \%)$ pneumonia (2 HAPs, 2 VAPs), three (15.8\%) ABSSSIs and one (5.3\%) PJI. Infections due to XDR-PA were polymicrobial in $6(31.6 \%)$ cases.

Septic shock was reported in $21.1 \%$ of the patients in the medical report.

Demographic and clinical characteristics of the patients selected are reported in Table 1.

Table 1. Demographic and clinical characteristics.

\begin{tabular}{|c|c|}
\hline Characteristics & Value $(n=19)$ \\
\hline Age in years, median (IQR) & $69(64-80)$ \\
\hline Male, $n(\%)$ & $13(68.4)$ \\
\hline \multicolumn{2}{|l|}{ Context of acquisition, $n(\%)$} \\
\hline Hospital acquired & $11(57.9)$ \\
\hline Healthcare-related & $8(42.1)$ \\
\hline Underlying diseases, $n(\%)$ & $19(100)$ \\
\hline Charlson comorbidity index, median (range) & $6(2-8)$ \\
\hline Chronic lung disease & $6(31.6)$ \\
\hline Solid malignancy & $5(26.3)$ \\
\hline Diabetes mellitus & $5(26.3)$ \\
\hline Chronic kidney disease & $4(21.1)$ \\
\hline Immunosuppression & $3(15.8)$ \\
\hline Trauma & $3(15.8)$ \\
\hline Hemodyalisis & $1(5.3)$ \\
\hline Prior surgery within 30 days, $n(\%)$ & $3(15.8)$ \\
\hline Prior invasive procedures within 30 days, $n(\%)$ & $5(26.3)$ \\
\hline Prior ICU stays within 90 days, $n(\%)$ & $5(26.3)$ \\
\hline Prior bacterial infections, $n(\%)$ & $16(84.2)$ \\
\hline Prior PA infections within 3 months & $5(26.3)$ \\
\hline Prior MDR/XDR pathogens, $n(\%)$ & $11(57.9)$ \\
\hline Prior antimicrobial use, within 90 days $n(\%)$ & $15(78.9)$ \\
\hline Carbapenem & $8(42.1)$ \\
\hline Glycopeptide & $8(42.1)$ \\
\hline Piperacillin/tazobactam & $7(36.8)$ \\
\hline Cephalosporin & $5(26.3)$ \\
\hline Colistin & $3(15.8)$ \\
\hline Fluoroquinolone & $3(15.8)$ \\
\hline Aminoglycoside & $2(10.5)$ \\
\hline Ceftazidime/avibactam & $1(5.3)$ \\
\hline Ceftolozane/tazobactam & $1(5.3)$ \\
\hline Other & $8(42.1)$ \\
\hline Sequential or combination use, $n(\%)$ & $12(63.2)$ \\
\hline Length of prior antimicrobial use $\geq 7$ days & $14(73.7)$ \\
\hline Indwelling device at infection onset, $n(\%)$ & $17(89.5)$ \\
\hline Urinary catheter & $14(73.7)$ \\
\hline Tracheostomy & $7(36.8)$ \\
\hline Central venous catheter & $5(26.3)$ \\
\hline Polymicrobial infection, $n(\%)$ & $6(31.6)$ \\
\hline \multicolumn{2}{|l|}{ Infection types, $n(\%)$} \\
\hline UTI & $6(31.6)$ \\
\hline BSI & $5(26.3)$ \\
\hline ABSSSI & $3(15.8)$ \\
\hline HAP & $2(10.5)$ \\
\hline VAP & $2(10.5)$ \\
\hline PJI & $1(5.3)$ \\
\hline
\end{tabular}

Pseudomonas aeruginosa, PA; Multidrug-resistant, MDR; ICU, intensive care unit; urinary tract infection, UTI bloodstream infections, BSI; Hospital-Acquired Pneumonia, HAP; Ventilator-Associated Pneumonia, VAP; acute bacterial skin and skin structure infections, ABSSIs; prosthetic joint infection, PJI.

Antimicrobial susceptibility of the tested isolates is listed in Table 2. Notably, all the isolates tested for colistin were susceptible. All the isolates were tested for ceftazidime/avibactam (C/A) and ceftolozane/tazobactam $(\mathrm{C} / \mathrm{T})$. Eight $(42.1 \%)$ were reported as susceptible to $\mathrm{C} / \mathrm{A}$ and seven $(36.8 \%)$ to $\mathrm{C} / \mathrm{T}$. Among the aminoglycoside class, six $(42.9 \%)$ out of 14 isolates tested for amikacin were susceptible, while none of the 
11 isolates tested for gentamicin was susceptible, three out of eight isolates tested for tobramycin were susceptible. Two out of $19(10.5 \%)$ tested isolates were susceptible to piperacillin/tazobactam and six out of 19 (31.6\%) to meropenem.

Table 2. Rates of antimicrobials' susceptibility.

\begin{tabular}{ccc}
\hline Antibiotics & No. Isolates Tested & Susceptibility $\boldsymbol{n}(\%)$ \\
\hline Ceftazidime & 19 & $3(15.8)$ \\
Cefepime & 19 & $1(5.3)$ \\
Ceftazidime/avibactam & 19 & $8(42.1)$ \\
Ceftolozane/tazobactam & 19 & $7(36.8)$ \\
Piperacillin/tazobactam & 19 & $2(10.5)$ \\
Meropenem & 19 & $6(31.6)$ \\
Imipenem & 11 & $3(27.3)$ \\
Ciprofloxacin & 19 & $1(5.3)$ \\
Colistin & 13 & $13(100)$ \\
Gentamicin & 11 & $0(0)$ \\
Amikacin & 14 & $6(42.9)$ \\
Tobramicin & 8 & $3(37.5)$
\end{tabular}

As reported in Table 3, empiric therapy was administered in 14 (73.7\%) of the patients, but it was appropriate only in two $(14.3 \%)$ cases. The time to start a target antibiotic therapy exceeded $48 \mathrm{~h}$ in $15(78.9 \%)$ cases. A targeted treatment consisted of anti-pseudomonal monotherapy in $12(63.2 \%)$ of the cases. In six cases, colistin monotherapy was administered based on antimicrobial susceptibility data, and in two of them, inhaled colistin was associated with pneumonia. The median duration of therapy was 10 days (IQR 7-14). Source control was performed in eight $(42.1 \%)$ of the cases. Clinical success was achieved in 17 (89.5\%) of the patients. Microbiologic failure was evaluated in 13 patients out of 19 through repeated cultures at the same site after at least seven days from the initiation of effective antibiotic therapy and reported in three patients (23.1\%). Two of them experimented clinical success later on, the other died due to a recurrent infection within 30 days.

One patient died due to a succeeding Candida bloodstream infection.

Table 3. Antibiotic therapies and outcomes.

\begin{tabular}{cc}
\hline Antibiotic Therapies and Outcome & Value $(\boldsymbol{n = 1 9 )}$ \\
\hline Empiric therapy, $n(\%)$ & $14(73.7)$ \\
Appropriate empiric therapy, $n(\%)$ & $2(10.5)$ \\
Start of a target therapy $>48 \mathrm{~h}, n(\%)$ & $15(78.9)$ \\
\hline Target monotherapy, $n(\%)$ & $12(63.2)$ \\
Colistin & $6(31.2)$ \\
Meropenem & $3(15.8)$ \\
Piperacillin/tazobactam & $1(5.3)$ \\
Amikacin & $1(5.3)$ \\
Ceftazidime/avibactam & $1(5.3)$ \\
Target Combination therapy, $n$ (\%) & $7(36.8)$ \\
Ceftazidime/Avibactam + Colistin & $2(15.4)$ \\
Colistin + Meropenem & $2(15.4)$ \\
Ceftolozane/tazobactam + Amikacin & $1(5.3)$ \\
Ceftazidime/avibactam + Amikacin & $1(5.3)$ \\
Colistin + Piperacillin/tazobactam & $1(5.3)$ \\
\hline Source control, $n(\%)$ & $8(42.1)$ \\
\hline Length of therapy in days, median (IQR) & $10(7-14)$ \\
\hline Death (30 days), $n(\%)$ & $2(15.4)$ \\
\hline Clinical success, $n(\%)$ & $17(89.5)$ \\
\hline Microbiological failure, $n /$ patients evaluated $(\%)$ & $3 / 13(23.1)$ \\
\hline Recurrent infection, $n(\%)$ & $1(5.3)$ \\
\hline
\end{tabular}




\section{Discussion}

Combined resistance to five antimicrobial groups is reported in PA around $3.4 \%$ in Europe [6]. Fewer data exist on the prevalence of XDR-PA [7,8]. Surprisingly, one Spanish survey reported that $17 \%$ of PA infections was due to XDR strains [1].

In our institution, the percentage of XDR-PA isolates on the total of the Pseudomonas aeruginosa isolates (n.609) from all sources from 1 June to 31 December 2020 is $3.1 \%$. This epidemiological result is in line with other previous reports [9].

Risk factors for XDR-PA have been exhaustively investigated in several studies and mostly addressed in prior hospital stay, previous antibiotic treatment (especially carbapenems and fluoroquinolones), prior ICU stay, previous PA colonization or infection, multiple comorbidities, and indwelling urinary catheter [10-17].

In our report, the majority of the patients had a median Age-Adjusted Charlson Comorbidity Index of 6 with at least one comorbidity, mostly chronic lung disease, diabetes, solid tumors, and chronic kidney disease. Among five patients with a previous PA infection/colonization, two were MDR/XDR (1/1, respectively). Previous PA isolation was consistent in our cohort, suggesting that a PA strain can build up resistance sequentially in the same patient.

Curiously, no patient was admitted to ICU at the time of the infection onset, even if $26.3 \%$ had reported a previous ICU stay. The infections developed mainly in medical wards such as Pneumology or high-intensity rehabilitation, where patients were often exposed to a unique combination of hazardous conditions for infections. A history of prior use of antibiotics was reported in the majority of the patients (78.9\%), with carbapenems $(42.1 \%)$ as the most frequent drug prescribed. The previous use of multiple antibiotics (in combinations or sequential over time) was present in $63.2 \%$ of the patients' history, possibly reflecting the importance of antimicrobial pressure on the development of resistance in PA.

For XDR-PA, polymyxin B, colistin, C/T, C/A, aztreonam, aminoglycosides and, recently, cefiderocol are the main therapeutic options depending on resistance profile. Combination therapy is not currently recommended. Treatment-emergent mutations conferring resistance to the new compounds are already reported [18-23].

In our case series, colistin was the only antibiotic to which $100 \%$ of tested isolates were susceptible. Further, we observed that $26.3 \%$ of our isolates were susceptible only to colistin. These data agree with others already reported in Southern European countries' literature, suggesting that colistin could represent the only therapeutic option available in these patients, although with some pharmacological and safety concerns [1,24-27].

Resistance to $\mathrm{C} / \mathrm{T}$ and/or $\mathrm{C} / \mathrm{A}$ is significant among the XDR-PA isolates selected. In literature, resistance is reported mainly due to carbapenemase production and some AmpC mutants. Other potential resistance mutations include specific large chromosomal deletions and PBP3 mutations, reduced membrane permeability and efflux pumps [20,28-31].

Even if the overall susceptibility rates of C/T against PA are high (94.1\% in Western Europe and $80.9 \%$ in Eastern Europe, respectively), the retained activity of this compound against meropenem non-susceptible PA isolates drops to $75.2 \%$ and $59.2 \%$ in Western and Eastern Europe, respectively. Even lower rates have been reported from Southern Asia $(37.9 \%)[30,32]$. Low susceptibility rates to $\mathrm{C} / \mathrm{T}$ in the subgroup of XDR-PA isolates have already been reported in Europe and the Middle East literature [33-35].

In our case series, probably due to the high percentage of resistance, $\mathrm{C} / \mathrm{T}$ has been rarely used in target therapy. In four cases, even if susceptibility to C/T was documented, meropenem was chosen as target therapy or continued if started as empiric therapy due to clinical improvement without an Infectious Disease specialist's consultation. In another case due to a strain susceptible to $\mathrm{C} / \mathrm{T}$, a UTI, amikacin was preferred to $\mathrm{C} / \mathrm{T}$ as target therapy due to the possibility of a single dose administration in a patient with serious difficulty in having stable venous access. In one case, C/A was adopted as a target therapy for a polymicrobial infection due to XDR-PA and Klebsiella pneumoniae carbapenemase-producing K. pneumoniae. 
In all but two cases, $\mathrm{C} / \mathrm{T}$ resistance was shared with $\mathrm{C} / \mathrm{A}$. The cross-resistance between the two compounds has already been documented [36].

Among the 19 isolates, those belonging to blood cultures (n.5) were resistant to both $\mathrm{C} / \mathrm{A}$ and $\mathrm{C} / \mathrm{T}$. All these isolates were tested retrospectively for the presence of carbapenemase enzymes and two out of five were found to harbor a carbapenemase VIM type. One respiratory tract sample PA-isolate, resistant to both the new drugs, was also evaluated for carbapenemases. An IMP-type carbapenemase was found. This finding of the prevalence of MBL in PA isolates has already been underlined in previous reports [37,38] and highlights that production of MBL carbapenemases is one of the main determinants leading to complex resistance profiles. While the development of resistance to $\mathrm{C} / \mathrm{T}$ and $\mathrm{C} / \mathrm{A}$ during treatment of PA infections is already concerning, in our case series the development of resistance to $\mathrm{C} / \mathrm{T}$ and $\mathrm{C} / \mathrm{A}$ was apparently not related to previous exposure to these drugs with the exception of one patient who had a recent history of exposure to both drugs at the time of the infection onset [23].

Consistent with other reports, among aminoglycosides, our data reveal that PA shows a higher susceptibility for amikacin then for other aminoglycosides (tobramycin and gentamicin) [39-41].

Even if empiric therapy was prescribed to the majority of the patients with XDR-PA infections in our case series it was rarely appropriate and a target therapy was usually not administered in the first $48 \mathrm{~h}$. In case of XDR profile it is highly unlikely that an appropriate empiric monotherapy can be chosen without knowledge of the isolate susceptibility.

Resistance in PA isolates has already been reported in other studies as an independent factor for an inappropriate initial antibiotic treatment, resulting in poor outcomes and increased costs. The antimicrobial susceptibility pattern of the infecting strain is obviously not available when empirical therapy is prescribed and an XDR profile is not usually taken into account outside the ICU and in patients who are not critically ill [42-49].

Due to the retrospective design of our study, the very small sample size and the inclusion of patients with different types of infections, no conclusive results about mortality can be deduced. However, it is remarkable that three $(23.1 \%)$ of the patients evaluated for microbiological failure with control cultures at the same site experienced the re-isolation of PA with the same resistance profile. Respiratory infections were found in $2 / 3$ of the cases in which microbiological eradication was not achieved. One of these patients experienced a recurrent XDR-PA infection with a fatal outcome. The worse outcome of lung infections has already been underlined, probably due to pharmacokinetic concerns of available drugs, infeasibility of adequate source control and, sometimes, more critical conditions [50].

\section{Conclusions}

Our data show that XDR-PA can be a threatening problem even outside the ICUs, especially in some wards that simulate the picture of long-term acute care hospitals: facilities specialized in the treatment of patients with serious medical conditions that no longer need intensive care but require more than they can receive in a rehabilitation center. Such frail patients can be more prone to acquire XDR-PA due to a combination of risky conditions (long hospital or previous ICU stay, multiple medical devices, previous multiple antibiotic treatments, particularly carbapenems, MDR colonization).

Despite their high susceptibility and good activity in PA, even MDR, the use of $\mathrm{C} / \mathrm{A}$ and $\mathrm{C} / \mathrm{T}$ in the contest of some XDR-PA strains could be seriously restricted due to reduced susceptibility.

Colistin, with highly preserved susceptibility, could often represent the only available therapeutic option against such isolates, but with significant safety concerns (kidney failure) and known efficacy limits, especially in some difficult-to-treat sources of infections (i.e., lung).

Thus, in settings where resistance to $\mathrm{C} / \mathrm{T}$ or $\mathrm{C} / \mathrm{A}$ is present, given the well-known colistin toxicity concern, cefiderocol could become an interesting perspective in the treatment of such XDR-PA infections. 
Knowing local resistance rates and geographical variation in new drugs' susceptibility due to the distinct local molecular epidemiology is essential in approaching XDR-PA severe infections.

Further, it seems crucial to have susceptibility testing results for all newer agents available as soon as possible.

Author Contributions: Conceptualization, F.R.; investigation, A.R.L. and B.F.; writing-original draft preparation, P.D.G.; supervision, M.T. All authors have read and agreed to the published version of the manuscript.

Funding: This research received no external funding.

Institutional Review Board Statement: The study was conducted according to the guidelines of the Declaration of Helsinki and approved by the Ethics Committee of the Fondazione Policlinico Universitario A. Gemelli IRCCS (protocol number 0040658/21).

Informed Consent Statement: Patient consent was waived due to retrospective observational design of the study.

Data Availability Statement: The data presented in this study are available on request from the corresponding author.

Conflicts of Interest: The authors declare no conflict of interest.

\section{References}

1. Del Barrio-Tofiño, E.; Zamorano, L.; Cortes-Lara, S.; López-Causapé, C.; Sánchez-Diener, I.; Cabot, G.; Bou, G.; Martínez-Martínez, L.; Oliver, A.; GEMARA-SEIMC/REIPI Pseudomonas Study Group. Spanish nationwide survey on Pseudomonas aeruginosa antimicrobial resistance mechanisms and epidemiology. J. Antimicrob. Chemother. 2019, 74, 1825-1835. [CrossRef] [PubMed]

2. Magiorakos, A.P.; Srinivasan, A.; Carey, R.B.; Carmeli, Y.; Falagas, M.E.; Giske, C.G.; Harbarth, S.; Hindler, J.F.; Kahlmeter, G.; Olsson-Liljequist, B.; et al. Multidrug-resistant, extensively drug-resistant and pandrug-resistant bacteria: An international expert proposal for interim standard definitions for acquired resistance. Clin. Microbiol. Infect. 2012, 18, 268-281. [CrossRef] [PubMed]

3. The European Committee on Antimicrobial Susceptibility Testing. Breakpoint Tables for Interpretation of MICs and Zone Diameters. Version 10.0. 2020. Available online: https://www.eucast.org/fileadmin/src/media/PDFs/EUCAST_files/Breakpoint_ tables/v_10.0_Breakpoint_Tables.pdf (accessed on 20 November 2021).

4. The European Committee on Antimicrobial Susceptibility Testing (EUCAST). Breakpoint tables for interpretation of MICs and Zone Diameters.Version 11.0. 2021. Available online: https://www.eucast.org/fileadmin/src/media/PDFs/EUCAST_files/ Breakpoint_tables/v_11.0_Breakpoint_Tables.pdf (accessed on 20 November 2021).

5. Giordano, L.; Fiori, B.; D’Inzeo, T.; Parisi, G.; Liotti, F.M.; Menchinelli, G.; De Angelis, G.; De Maio, F.; Luzzaro, F.; Sanguinetti, M.; et al. Simplified Testing Method for Direct Detection of Carbapenemase-Producing Organisms from Positive Blood Cultures Using the NG-Test Carba 5 Assay. Antimicrob. Agents Chemother. 2019, 63, e00550-19. [CrossRef] [PubMed]

6. European Centre for Disease Prevention and Control. Antimicrobial resistance in the EU/EEA (EARS-Net)-Annual Epidemiological Report 2019. Stockholm: ECDC. 2020. Available online: https:/ /www.ecdc.europa.eu/sites/default/files/documents/ surveillance-antimicrobial-resistance-Europe-2019.pdf (accessed on 29 November 2021).

7. WHO Regional Office for Europe and European Centre for Disease Prevention and Control. Surveillance of Antimicrobial Resistance in Europe, 2020 Data. Executive Summary. Copenhagen: WHO Regional Office for Europe. 2021. Available online: https:/ / www.ecdc.europa.eu/sites/default/files/documents/Surveillance-antimicrobial-resistance-in-Europe-2020.pdf (accessed on 29 November 2021).

8. Castanheira, M.; Deshpande, L.M.; Costello, A.; Davies, T.A.; Jones, R.N. Epidemiology and carbapenem resistance mechanisms of carbapenem-non-susceptible Pseudomonas aeruginosa collected during 2009-11 in 14 European and Mediterranean countries. J. Antimicrob. Chemother. 2014, 69, 1804-1814. [CrossRef] [PubMed]

9. Kadri, S.S.; Adjemian, J.; Lai, Y.L.; Spaulding, A.B.; Ricotta, E.; Prevots, D.R.; Palmore, T.N.; Rhee, C.; Klompas, M.; Dekker, J.P.; et al. National Institutes of Health Antimicrobial Resistance Outcomes Research Initiative (NIH-ARORI). Difficult-to-Treat Resistance in Gram-negative Bacteremia at 173 US Hospitals: Retrospective Cohort Analysis of Prevalence, Predictors, and Outcome of Resistance to All First-line Agents. Clin. Infect. Dis. 2018, 67, 1803-1814. [CrossRef] [PubMed]

10. Burillo, A.; Muñoz, P.; Bouza, E. Risk stratification for multidrug-resistant Gram-negative infections in ICU patients. Curr. Opin. Infect. Dis. 2019, 32, 626-637. [CrossRef]

11. Raman, G.; Avendano, E.E.; Chan, J.; Merchant, S.; Puzniak, L. Risk factors for hospitalized patients with resistant or multidrugresistant Pseudomonas aeruginosa infections: A systematic review and meta-analysis. Antimicrob. Resist. Infect. Control 2018, 7, 79. [CrossRef]

12. Bodro, M.; Sabé, N.; Tubau, F.; Lladó, L.; Baliellas, C.; González-Costello, J.; Cruzado, J.M.; Carratalà, J. Extensively drug-resistant Pseudomonas aeruginosa bacteremia in solid organ transplant recipients. Transplantation 2015, 99, 616-622. [CrossRef] 
13. Willmann, M.; Klimek, A.M.; Vogel, W.; Liese, J.; Marschal, M.; Autenrieth, I.B.; Peter, S.; Buhl, M. Clinical and treatment-related risk factors for nosocomial colonisation with extensively drug-resistant Pseudomonas aeruginosa in a haematological patient population: A matched case control study. BMC Infect. Dis. 2014, 14, 650. [CrossRef]

14. Samonis, G.; Vardakas, K.Z.; Kofteridis, D.P.; Dimopoulou, D.; Andrianaki, A.M.; Chatzinikolaou, I.; Katsanevaki, E.; Maraki, S.; Falagas, M.E. Characteristics, risk factors and outcomes of adult cancer patients with extensively drug-resistant Pseudomonas aeruginosa infections. Infection 2014, 42, 721-728. [CrossRef]

15. Bassetti, M.; Righi, E.; Vena, A.; Graziano, E.; Russo, A.; Peghin, M. Risk stratification and treatment of ICU-acquired pneumonia caused by multidrug-resistant/extensively drug-resistant/pandrug-resistant bacteria. Curr. Opin. Crit. Care 2018, 24, 385-393. [CrossRef] [PubMed]

16. Trinh, T.D.; Zasowski, E.J.; Claeys, K.C.; Lagnf, A.M.; Kidambi, S.; Davis, S.L.; Rybak, M.J. Multidrug-resistant Pseudomonas aeruginosa lower respiratory tract infections in the intensive care unit: Prevalence and risk factors. Diagn. Microbiol. Infect. Dis. 2017, 89, 61-66. [CrossRef] [PubMed]

17. Tartof, S.Y.; Kuntz, J.L.; Chen, L.H.; Wei, R.; Puzniak, L.; Tian, Y.; Im, T.M.; Takhar, H.S.; Merchant, S.; Lodise, T. Development and Assessment of Risk Scores for Carbapenem and Extensive $\beta$-Lactam Resistance Among Adult Hospitalized Patients with Pseudomonas aeruginosa Infection. JAMA Netw. Open 2018, 1, e183927. [CrossRef] [PubMed]

18. Horcajada, J.P.; Montero, M.; Oliver, A.; Sorlí, L.; Luque, S.; Gómez-Zorrilla, S.; Benito, N.; Grau, S. Epidemiology and Treatment of Multidrug-Resistant and Extensively Drug-Resistant Pseudomonas aeruginosa Infections. Clin. Microbiol. Rev. 2019, 32, e00031-19. [CrossRef] [PubMed]

19. Haidar, G.; Philips, N.J.; Shields, R.K.; Snyder, D.; Cheng, S.; Potoski, B.A.; Doi, Y.; Hao, B.; Press, E.G.; Cooper, V.S.; et al. Ceftolozane-Tazobactam for the Treatment of Multidrug-Resistant Pseudomonas aeruginosa Infections: Clinical Effectiveness and Evolution of Resistance. Clin. Infect. Dis. 2017, 65, 110-120. [CrossRef]

20. Pogue, J.M.; Kaye, K.S.; Veve, M.P.; Patel, T.S.; Gerlach, A.T.; Davis, S.L.; Puzniak, L.A.; File, T.M.; Olson, S.; Dhar, S.; et al. Ceftolozane/Tazobactam vs. Polymyxin or Aminoglycoside-based Regimens for the Treatment of Drug-resistant Pseudomonas aeruginosa. Clin. Infect. Dis. 2020, 71, 304-310. [CrossRef]

21. Giacobbe, D.R.; Bassetti, M.; De Rosa, F.G.; Del Bono, V.; Grossi, P.A.; Menichetti, F.; Pea, F.; Rossolini, G.M.; Tumbarello, M.; Viale, P.; et al. Ceftolozane/tazobactam: Place in therapy. Expert Rev. Anti. Infect. Ther. 2018, 16, 307-320. [CrossRef]

22. Bassetti, M.; Castaldo, N.; Cattelan, A.; Mussini, C.; Righi, E.; Tascini, C.; Menichetti, F.; Mastroianni, C.M.; Tumbarello, M.; Grossi, P.; et al. Ceftolozane/tazobactam for the treatment of serious Pseudomonas aeruginosa infections: A multicentre nationwide clinical experience. Int. J. Antimicrob. Agents 2019, 53, 408-415. [CrossRef]

23. Rubio, A.M.; Kline, E.G.; Jones, C.E.; Chen, L.; Kreiswirth, B.N.; Nguyen, M.H.; Clancy, C.J.; Cooper, V.S.; Haidar, G.; Van Tyne, D.; et al. In vitro Susceptibility of Multidrug-Resistant Pseudomonas aeruginosa following Treatment-emergent Resistance to Ceftolozane-tazobactam. Antimicrob. Agents Chemother. 2021, 65, e00084-21. [CrossRef]

24. Pérez, A.; Gato, E.; Pérez-Llarena, J.; Fernández-Cuenca, F.; Gude, M.J.; Oviaño, M.; Pachón, M.E.; Garnacho, J.; González, V.; Pascual, Á.; et al. High incidence of MDR and XDR Pseudomonas aeruginosa isolates obtained from patients with ventilatorassociated pneumonia in Greece, Italy and Spain as part of the MagicBullet clinical trial. J. Antimicrob. Chemother. 2019, 74, 1244-1252. [CrossRef]

25. Del Barrio-Tofiño, E.; López-Causapé, C.; Cabot, G.; Rivera, A.; Benito, N.; Segura, C.; Montero, M.M.; Sorlí, L.; Tubau, F.; Gómez-Zorrilla, S.; et al. Genomics and Susceptibility Profiles of Extensively Drug-Resistant Pseudomonas aeruginosa Isolates from Spain. Antimicrob. Agents Chemother. 2017, 61, e01589-17. [CrossRef] [PubMed]

26. Fournier, D.; Carrière, R.; Bour, M.; Grisot, E.; Triponney, P.; Muller, C.; Lemoine, J.; Jeannot, K.; Plésiat, P.; GERPA Study Group. Mechanisms of Resistance to Ceftolozane/Tazobactam in Pseudomonas aeruginosa: Results of the GERPA Multicenter Study. Antimicrob. Agents Chemother. 2021, 65, e01117-20. [CrossRef] [PubMed]

27. Giacobbe, D.R.; Saffioti, C.; Losito, A.R.; Rinaldi, M.; Aurilio, C.; Bolla, C.; Boni, S.; Borgia, G.; Carannante, N.; Cassola, G.; et al SITA GIOVANI (Young Investigators Group of the Società Italiana Terapia Antinfettiva) and the COLI-CROSS Study Group. Use of colistin in adult patients: A cross-sectional study. J. Glob. Antimicrob. Resist. 2020, 20, 43-49. [CrossRef]

28. Winkler, M.L.; Papp-Wallace, K.M.; Hujer, A.M.; Domitrovic, T.N.; Hujer, K.M.; Hurless, K.N.; Tuohy, M.; Hall, G.; Bonomo, R.A. Unexpected challenges in treating multidrug-resistant Gram-negative bacteria: Resistance to ceftazidime-avibactam in archived isolates of Pseudomonas aeruginosa. Antimicrob. Agents Chemother. 2015, 59, 1020-1029. [CrossRef] [PubMed]

29. Skoglund, E.; Abodakpi, H.; Rios, R.; Diaz, L.; De La Cadena, E.; Dinh, A.Q.; Ardila, J.; Miller, W.R.; Munita, J.M.; Arias, C.A.; et al. In Vivo Resistance to Ceftolozane/Tazobactam in Pseudomonas aeruginosa Arising by AmpC-and Non-AmpC-Mediated Pathways. Case Rep. Infect. Dis. 2018, 9095203. [CrossRef] [PubMed]

30. Teo, J.Q.; Lim, J.C.; Tang, C.Y.; Lee, S.J.; Tan, S.H.; Sim, J.H.; Ong, R.T.; Kwa, A.L. Ceftolozane/Tazobactam Resistance and Mechanisms in Carbapenem-Nonsusceptible Pseudomonas aeruginosa. mSphere 2021, 6, e01026-20. [CrossRef]

31. Cabot, G.; Rodrigo, E.; Sánchez-Diener, I.; Zamorano, L.; Oliver, A. Poster P1173-Mechanisms of Pseudomonas aeruginosa betalactam resistance development independent of beta-lactamase production. In Proceedings of the Poster Session 058. Diverse Mechanisms of Resistance-Gram-Negative, 28th ECCMID, Madrid, Spain, 22 April 2018.

32. Sader, H.S.; Carvalhaes, C.G.; Duncan, L.R.; Flamm, R.K.; Shortridge, D. Susceptibility trends of ceftolozane/tazobactam and comparators when tested against European Gram-negative bacterial surveillance isolates collected during 2012-18. J. Antimicrob. Chemother. 2020, 75, 2907-2913. [CrossRef] 
33. Sader, H.S.; Duncan, L.R.; Doyle, T.B.; Castanheira, M. Antimicrobial activity of ceftazidime/avibactam, ceftolozane/tazobactam and comparator agents against Pseudomonas aeruginosa from cystic fibrosis patients. JAC Antimicrob. Resist. 2021, 3 , dlab126. [CrossRef]

34. Sader, H.S.; Carvalhaes, C.G.; Shortridge, D.; Castanheira, M. Comparative activity of newer $\beta$-lactam/ $\beta$-lactamase inhibitor combinations against Pseudomonas aeruginosa from patients hospitalized with pneumonia in European medical centers in 2020. Eur. J. Clin. Microbiol. Infect. Dis. 2021, 41, 319-324. [CrossRef]

35. Sader, H.S.; Farrell, D.J.; Castanheira, M.; Flamm, R.K.; Jones, R.N. Antimicrobial activity of ceftolozane/tazobactam tested against Pseudomonas aeruginosa and Enterobacteriaceae with various resistance patterns isolated in European hospitals (2011-12). J. Antimicrob. Chemother. 2014, 69, 2713-2722. [CrossRef]

36. Arca-Suárez, J.; Lasarte-Monterrubio, C.; Rodiño-Janeiro, B.K.; Cabot, G.; Vázquez-Ucha, J.C.; Rodríguez-Iglesias, M.; GalánSánchez, F.; Beceiro, A.; González-Bello, C.; Oliver, A.; et al. Molecular mechanisms driving the in vivo development of OXA-10-mediated resistance to ceftolozane/tazobactam and ceftazidime/avibactam during treatment of XDR Pseudomonas aeruginosa infections. J. Antimicrob. Chemother. 2021, 76, 91-100. [CrossRef] [PubMed]

37. Del Barrio-Tofiño, E.; López-Causapé, C.; Oliver, A. Pseudomonas aeruginosa epidemic high-risk clones and their association with horizontally-acquired $\beta$-lactamases: 2020 update. Int. J. Antimicrob. Agents 2020, 56, 106196. [CrossRef] [PubMed]

38. Woodworth, K.R.; Walters, M.S.; Weiner, L.M.; Edwards, J.; Brown, A.C.; Huang, J.Y.; Malik, S.; Slayton, R.B.; Paul, P.; Capers, C.; et al. Vital Signs: Containment of Novel Multidrug-Resistant Organisms and Resistance Mechanisms-United States, $2006-2017$. MMWR Morb. Mortal. Wkly. Rep. 2018, 67, 396. [CrossRef] [PubMed]

39. Kantor, R.J.; Norden, C.W. In vitro activity of netilmicin, gentamicin, and amikacin. Antimicrob. Agents Chemother. 1977, 11, 126-131. [CrossRef] [PubMed]

40. Castanheira, M.; Davis, A.P.; Mendes, R.E.; Serio, A.W.; Krause, K.M.; Flamm, R.K. In vitro activity of plazomicin against gram-negative and gram-positive isolates collected from U.S. hospitals and comparative activities of aminoglycosides against carbapenem-resistant Enterobacteriaceae and isolates carrying carbapenemase genes. Antimicrob. Agents Chemother. 2018, 62, e00313-18. [CrossRef]

41. Nichols, W.W.; de Jonge, B.L.; Kazmierczak, K.M.; Karlowsky, J.A.; Sahm, D.F. In Vitro Susceptibility of Global Surveillance Isolates of Pseudomonas aeruginosa to Ceftazidime-Avibactam (INFORM 2012 to 2014). Antimicrob. Agents Chemother. 2016, 60, 4743-4749. [CrossRef]

42. Savage, R.D.; Fowler, R.A.; Rishu, A.H.; Bagshaw, S.M.; Cook, D.; Dodek, P.; Hall, R.; Kumar, A.; Lamontagne, F.; Lauzier, F. The Effect of Inadequate Initial Empiric Antimicrobial Treatment on Mortality in Critically Ill Patients with Bloodstream Infections: A Multi-Centre Retrospective Cohort Study. PLoS ONE 2016, 11, e0154944. [CrossRef]

43. Lambregts, M.M.C.; Wijnakker, R.; Bernards, A.T.; Visser, L.G.; Cessie, S.L.; Boer, M.G.J. Mortality after Delay of Adequate Empiric Antimicrobial Treatment of Bloodstream Infection. J. Clin. Med. 2020, 9, 1378. [CrossRef]

44. Tumbarello, M.; De Pascale, G.; Trecarichi, E.M.; Spanu, T.; Antonicelli, F.; Maviglia, R.; Pennisi, M.A.; Bello, G.; Antonelli, M. Clinical outcomes of Pseudomonas aeruginosa pneumonia in intensive care unit patients. Intensive Care Med. 2013, 39, 682-692. [CrossRef]

45. Luu, Q.; Vitale, K.; Shan, G.; Jayakumar, R.; Viswesh, V. Evaluation of Guideline Recommendations for Dual Antipseudomonal Therapy in Hospitalized Adults with Pneumonia Using Combination Antibiograms. Pharmacotherapy 2020, 40, 1089-1098. [CrossRef]

46. Liu, Q.; Li, X.; Li, W.; Du, X.; He, J.Q.; Tao, C.; Feng, Y. Influence of carbapenem resistance on mortality of patients with Pseudomonas aeruginosa infection: A meta-analysis. Sci. Rep. 2015, 5, 11715. [CrossRef] [PubMed]

47. Barrasa-Villar, J.I.; Aibar-Remón, C.; Prieto-Andrés, P.; Mareca-Doñate, R.; Moliner-Lahoz, J. Impact on Morbidity, Mortality, and Length of Stay of Hospital-Acquired Infections by Resistant Microorganisms. Clin. Infect. Dis. 2017, 65, 644-652. [CrossRef] [PubMed]

48. Righi, E.; Peri, A.M.; Harris, P.N.; Wailan, A.M.; Liborio, M.; Lane, S.W.; Paterson, D.L. Global prevalence of carbapenem resistance in neutropenic patients and association with mortality and carbapenem use: Systematic review and meta-analysis. J. Antimicrob. Chemother. 2017, 72, 668-677. [CrossRef] [PubMed]

49. Riu, M.; Chiarello, P.; Terradas, R.; Sala, M.; Garcia-Alzorriz, E.; Castells, X.; Grau, S.; Cots, F. Cost Attributable to Nosocomial Bacteremia. Analysis According to Microorganism and Antimicrobial Sensitivity in a University Hospital in Barcelona. PLoS ONE 2016, 11, e0153076. [CrossRef] [PubMed]

50. Shields, R.K.; Nguyen, M.H.; Chen, L.; Press, E.G.; Kreiswirth, B.N.; Clancy, C.J. Pneumonia and renal replacement therapy are risk factors for ceftazidime-avibactam treatment failures and resistance among patients with carbapenem-resistant Enterobacteriaceae infections. Antimicrob. Agents Chemother. 2018, 62, e02497-17. [CrossRef] 